\title{
The Test-Retest Reliability and Minimal Detectable Change of the Fugl-Meyer Assessment of the Upper Extremity and 9-Hole Pegboard Test in Individuals With Subacute Stroke
}

\author{
Sahar Toluee Achacheluee ${ }^{1}$, Leila Rahnama ${ }^{1 *}$, Nouredin Karimi ${ }^{1}$, Iraj Abdollahi ${ }^{1}$, Shapour Jaberzadeh ${ }^{2}$, Syed Asadullah Arslan $^{3}$ \\ 1. Department of Physical Therapy, University of Social Welfare and Rehabilitation Sciences, Tehran, Iran. \\ 2. Department of Physical Therapy, Faculty of Medicine, Monash University, Melbourne, Australia. \\ 3. Department of Physiotherapy, School of Rehabilitation, International Campus, Tehran University of Medical Sciences, Tehran, Iran.
}

Citation: Toluee Achacheluee S, Rahnama L, Karimi N, Abdollahi I, Jaberzadeh Sh, Arslan SA. The Test-Retest Reliability and Minimal Detectable Change of the Fugl-Meyer Assessment of the Upper Extremity and 9-Hole Pegboard Test in Individuals With Subacute Stroke. Physical Treatments. 2016; 5(4):225-230.

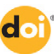

Article info:

Received: 01 Sep. 2015

Accepted: 02 Dec. 2015
Keywords:

Stroke, Reliability, FuglMeyer, 9-hole pegboard, Test-Retest

\begin{abstract}
A B S T RA C T
Purpose: The first step to manage motor impairment of upper limb in patients with subacute stroke is having an accurate assessment tool. The Fugl-Meyer assessment of upper extremity and 9-hole pegboard test are used to evaluate motor function and hand dexterity in stroke survivals. The present study aimed to investigate the test-retest reliability and minimal detectable change (MDC) in these two tests.
\end{abstract}

Methods: A total of 15 patients with subacute stroke (54-76 years old) participated in this study. They were selected non-randomly from rehabilitation clinics and hospitals of Tehran, Iran, based on inclusive and exclusive criteria. Intraclass correlation coefficient (ICC), standard error measurement (SEM), and MDC were used for investigating intraday and interday reliability for 1 hour and 3 days.

Results: Intraday reliabilities of Fugl-Meyer and 9-hole pegboard were excellent with ICC of 0.98 and 0.98 , also MDC of 1.96 and 8.59, respectively. The interday reliabilities of these tests were also excellent with ICC of 0.99 and 0.96 , as well as MDC of 1.52 and 12.69 , respectively. The absolute reliability (SEM) was less than $10 \%$ of maximum acquired scores indicating acceptable errors of measurement.

Conclusion: Results show that the Fugl-Meyer assessment and 9-hole pegboard test have excellent test-retest reliability. Therefore these tests can be used for appropriate treatment planning and clinical decision making in patients with subacute stroke.

\section{Introduction}

troke is a major cause of disability among elders in the world and affects mostly the upper extremity [1]. Upper limb disability does not recover in $50 \%$ of cases after stroke [2]. Almost 20\% to $30 \%$ patients with stroke remain dependent even after recent progress in the treatment of patients with acute

\section{* Corresponding Author:}

Leila Rahnama, PhD

Address: Department of Physiotherapy, University of Social Welfare and Rehabilitation Sciences, Kodakyar Ave., Daneshjo Blvd., Evin, Tehran, Iran. Phone: +98 (21) 22180039

E-mail:lrahnama@gmail.com 
and chronic stroke [3]. Recovery phase is fast during 6 months after stroke due to reconstruction process of brain and neuroplasticity. Therefore, this period is important for treatment tasks [4]. After stroke, anticipatory changes in the motor control of upper limb are the most important strategies for rehabilitation [5]. Selection of suitable measurement test should be according to level and framework of International Classification of Functioning, Disability, and Health (ICF) [6]. All selected measuring tests should have psychometric properties and one of the most important properties is reliability [7].

Test-retest measurement of upper limb function in patients with stroke is essential for better treatment choices, making clinical decisions, and helps in future research. Obtaining test-retest reliability for the function of upper limb is important both for clinic and research. Fugl-Meyer assessment (FMA) and 9-hole pegboard test (9-HPT) are the two most popular assessment tools for upper extremity. FMA is used to evaluate and measure upper limb recovery in post-stroke hemiplegic patients while 9-HPT is used as a simple, quick assessment for finger $[8,9]$.

Previously, test-retest reliability of the above mentioned tests had not been determined perfectly. For example, inter- and intra-reliability of examiners for FMA on patients with stroke was better performed, however most study patients were in chronic phase. So, it lacked the explanation about acute phase $[10,11]$. In another study, test-retest reliability was seriously done for 9-HPT but the participants were healthy population $[12,13]$.

Patients with stroke have different characteristics at each stage of rehabilitation (acute: less than 1 month, subacute: 1 to 6 months, chronic: more than 6 months) that may have different effects on the motor functional measurement [14$16]$. Because the recovery process is automatic during subacute phase [14], the therapists must identify whether rehabilitation helps the recovery or it happens naturally due to physiological process. For this reason, the present study investigates if the tests used to assess the movement of patients with stroke during subacute phase have satisfactory reliabilities and tries to relate the observed recovery during measured tests with the treatment, instead of natural recovery. The purpose of the present study was to assess the intraday and interday reliability of FMA and 9-HPT.

\section{Materials and Methods}

\section{Sample selection}

This non-experimental study was designed to investigate the reliability of FMA and 9-HPT. Fifteen patients with subacute stroke, aged between 53 and 76 years were selected from 4 rehabilitation centers in Tehran from July 2015 to December 2015. All patients who participated in this research signed consent forms and their information was kept confidential. The present study was approved by the Ethics Committee of University of Social Welfare and Rehabilitation Sciences.

The inclusion criteria were having first ischemic stroke with duration of 1 to 6 months, not treated with psychotherapeutic drugs that may change the cognitive status of the person (e.g. antidepressants and diazepam), having good alertness level to interact with the examiner and understand exam contents (patients should get 23 points from Mini-Mental Status Examination [MMSE] performed by examiner) [17], not being under any rehabilitation therapy during study.

The exclusion criteria were having any other neurological disease, having motor disability in upper extremity, particularly any deformity and limited range of motion (ROM) in upper limb which may cause difficulty during task performance, and lack of cooperation by patient during test.

\section{Study tools}

Data collection tools comprised Demographic questionnaire for general information, Fugl-Meyer assessment (FMA), and 9-Hole Peg Test (9-HPT). FMA, which quantitatively measures the level of sensorimotor impairment in patients with hemiplegic stroke was developed in 1975 [18]. This scale has 5 assessment recovery domains, which evaluate motor functioning of the upper and lower extremities, balance, sensation, joint range of motion, and joint pain in post-stroke patients. Part of the test related to upper limb has 33 items with total score of 66. Each task is scored from 0 to 2, with 0 stating inability of the individual to do the task and 2 representing that patient can fully accomplish the task. The 9-HPT is a standardized, quantitative test used for the assessment of motor skills, speed, and accuracy of hands [19]. Assessment tools consists of a rectangular board with 9 holes in 3 rows. Another rectangular board located above also has 9 holes with 9 pegs in the holes. The participants were instructed to pick up each peg, one by one, as quickly as possible and place them in the 9 holes in the board below. Total duration of the task is recorded by chronometer and registered as score of the individual.

\section{Procedure}

Intraday reliability of FMA and 9-HPT were done with one hour interval while interday reliability of these tests were repeated with the interval of 9 days. The examiner 
and the chronometer were similar in all tests. Intraday and interday reliability of both tests were analyzed using correlation coefficient with SEM (standard error of measurement) and ICC (Intraclass correlation coefficient). According to the classification of Duncan et al., ICC between 0.50 and 0.69 was considered as average reliability, between 0.70 and 0.89 as high, and between 0.90 and 1 as very high reliable [11]. If the amount of SEM is less than $10 \%$ of maximum scores, then it shows the desirable level of absolute reliability and if it is more than $10 \%$, then it is not a desirable scale of absolute reliability [20,21].

\section{Results}

Table 1 presents the demographic characteristics of the participants. An examiner administered FMA and 9-HPT and the obtained ICC, minimal detectable change (MDC), standard error of measurement (SEM) were used to assess the relative and absolute reliabilities of the tests.

Tables 2 and 3 indicated that the ICC value of intraday with 1 hour interval in FMA was 0.98 and in 9-HPT was also 0.98 , while ICC value of interday for FMA was 0.99 and for 9-HPT was 0.96. SEM in intraday for FMA was 0.71 and for 9-HPT was 3.10. SEM in interday for FMA was 0.55 and for $9-\mathrm{HPT}$ was 4.58. Also, MDC in intraday for FMA was 1.96 and for 9-HPT was 8.59 while interday for FMA was 1.52 and for 9-HPT was 12.69.

\section{Discussion}

Daily motor functions of every individual mostly depend on the function of upper limb [22]. Stroke effects disturb the daily activities of the patients and upper limb disorder causes anxiety as well as poor understanding of the surroundings $[23,24]$. Therefore, recovery of upper limb is the most important part of rehabilitation after stroke [25]. For a better treatment, detailed assessment of motor function should be performed. In this regard, evaluation of available assessment tests for upper limb function of the patients with stroke is essential. Use of highly reliable tools and tests minimizes the doubts regarding disease and provides better treatment choices [26].

The aim of the intraday and interday reliability of FMA and 9-HPT was to assess motor function and manual dexterity of patients with subacute stroke. Results reported that intraday and interday reliability of both tests was high for the assessment of motor function and manual dexterity in these patients. SEM score was less than $10 \%$ of the maximum obtained score.

According to previous studies, this intraday and interday reliability had high confidence interval (95\% CI) [27-30]. Stanford et al. assessed within examiner reliability among 3 therapists in a day by using the original version of FMA and reported the ICC as 0.97 which is

Table 1. Characteristics of study patients.

\begin{tabular}{cccc}
\hline & Age, $y$ & Time After Stroke (WK) & MMSE \\
\hline Mean $\pm S D$ & $47.64 \pm 6.76$ & $14.93 \pm 5.27$ & $27.4 \pm 1.5$ \\
\hline
\end{tabular}

PHYSICAL TREA T MENTS

Table 2. Intraday reliability.

\begin{tabular}{cccccc}
\hline & ICC & Lower Confidence Interval 95\% & Absolute SEM & Relative SEM, \% & MDC \\
\hline Fugl meyer & 0.98 & 0.96 & 0.71 & 1.8 & 1.96 \\
9-Hole pegboard & 0.98 & 0.91 & 3.1 & 4.39 & 8.59 \\
\hline
\end{tabular}

PHYSICAL TREA T MENTS

Table 3. Interday reliability.

\begin{tabular}{ccccc} 
& ICC & Lower Confidence Interval 95\% & Absolute SEM & Relative SEM, \% \\
\hline Fugl meyer & 0.99 & 0.99 & 0.55 & 1.43 \\
9-Hole pegboard & 0.96 & 0.91 & 4.58 & 6.39 \\
\hline
\end{tabular}


similar to the present study. But that test was performed just in one day which could not be applicable for patients with subacute stroke where automatic recovery due to neuroplasticity occurs over time [2, 5]. Michaelsen et al. [32] performed the inter-rater reliability of the FMA on patients with chronic stroke. They had similar results, i.e., ICC $=0.98$ with $\mathrm{CI}$ : $95 \%$ (99-94) like the present study, but did not mention SEM or less error of their research. Clinically, SEM is more satisfactory and helps in better decision making. Platz et al. [33] performed interday reliability of the FMA for upper limb on patients with multiple sclerosis (MS), stroke and concussion with 1 week interval. That study revealed an ICC of 0.97 and SEM of 3.6 which was almost similar to the results of the present study but that study was performed on patients with MS, stroke and concussion and could not be applied accurately just on patients with stroke. Another study by Kim et al. [10] was performed on patients with stroke to assess the test-retest reliability of FMA. It yielded similar results to the present study ( $\mathrm{ICC}=0.97,95 \% \mathrm{CI}$ : [94-98], SEM=3.2). Karimi et al. performed reliability between two examiners in FMA on patients with stroke and showed similar results with $\mathrm{ICC}=0.97$.

As mentioned earlier, recovery process at subacute phase is faster compared to chronic phase which may affect the assessment of motor function. Lundquist et al. [35] also tested the reliability between two examiners using FMA on patients with stroke at acute and subacute phases and got results $(\mathrm{ICC}=0.95)$ which were similar to the present study. The 9-Hole Peg test, used to assess the manual skills had the participants from 3 to 85 years old [36]. As the study had no intervention during test-retest duration, the results are expected to have no noticeable change. Obtained values in the present study had not shown any significant change for manual dexterity in patients with subacute stroke. The relative reliability of 9-HPT had shown much needed reliability. Results of the absolute reliability or SEM were less than $10 \%$ of obtained score and acquired error was acceptable. Results of the present and other studies indicated that 9-HPT for manual dexterity could be used in different conditions [37-39] and it showed acceptable ability to assess manual dexterity in patients with stroke.

Grice et al. [13] performed test-retest and intra-rater 9-HPT reliability. Intra-rater examiner showed high reliability $(\mathrm{ICC}=0.98)$ but test-retest showed moderate reliability $(\mathrm{ICC}=0.45)$. Their study had participants of different ages from 21 to 71 years that might be the reason for low reliability. Poole et al. [40] assessed 9-HPT testretest reliability on 5 to 10 years old children and found high reliability for 9-HPT. Backman et al. [41] during assessment of test-retest reliability of 9-HPT in differ- ent age groups found that older individuals ( $70-79$ years) showed high reliability compared to younger ones. At the end, we can conclude that FMA and 9-HPT has high reliability which could be trusted for the assessment of patients with subacute stroke.

Based on the results of present study, we suggest that similar studies with larger samples be performed to assess reliability of every part of the tests. According to previous studies, there was difference between two sexes during 9-HPT [12], so we also recommend that studies be done to find out sex differences during 9-HPT assessment.

\section{Acknowledgements}

The authors are very thankful to participants who attended in this research. We also express our sincere thanks to the staff and research assistants of Rofeideh Hospital, University of Social Welfare and Rehabilitation Sciences, Tehran, Iran who cooperate in patients' recruitment.

\section{Conflict of Interest}

The authors declared no conflict of interests.

\section{Reference}

[1] World Health Organization. The 10 leading causes of death in the world, 2000 and 2012. Geneva: World Health Organization; 2014

[2] Hendricks HT, van Limbeek J, Geurts AC, Zwarts MJ. Motor recovery after stroke: a systematic review of the literature Archives of Physical Medicine and Rehabilitation. 2002; 83(11):1629-637. doi: 10.1053/apmr.2002.35473

[3] Hankey G. Preventable stroke and stroke prevention. Journal of Thrombosis and Haemostasis. 2005; 3(8):1638-645. doi 10.1111/j.1538-7836.2005.01427.x

[4] Krakauer JW. Arm function after stroke: from physiology to recovery. Seminars in Neurology. 2005; 25(4):384-95. doi: $10.1055 / \mathrm{s}-2005-923533$

[5] De Weerdt W, Lincoln N, Harrison M. Prediction of arm and hand function recovery in stroke patients. International Journal of Rehabilitation Research. 1987; 10:110-12. doi: 10.1097/00004356-198700105-00022

[6] Jørgensen H, Nakayama H, Pedersen P, Kammersgaard L, Raaschou H, Olsen T. Epidemiology of stroke-related disability. Clinics in Geriatric Medicine. 1999; 15(4):785-99.

[7] Duncan P. Measuring recovery of function after stroke: clinical and measurement issues in selecting stroke outcome measures in clinical trials. Restorative neurology: advances in pharmacotherapy for recovery after stroke. New York: Futura Publishing; 1998. 
[8] Gladstone DJ, Danells CJ, Black SE. The Fugl-Meyer assessment of motor recovery after stroke: a critical review of its measurement properties. Neurorehabilitation and Neural Repair. 2002; 16(3):232-40. doi: 10.1177/154596802401105171

[9] Mathiowetz V, Weber K, Kashman N, Volland G. Adult norms for the nine hole peg test of finger dexterity. Occupation, Participation and Health. 1985; 5(1):24-38. doi: $10.1177 / 153944928500500102$

[10] Kim H, Her J, Ko J, Park DS, Woo JH, You Y, et al. Reliability, concurrent validity, and responsiveness of the FuglMeyer Assessment (FMA) for hemiplegic patients. Journal of Physical Therapy Science. 2012; 24(9):893-99. doi: 10.1589/ jpts.24.893

[11] Duncan PW, Propst M, Nelson SG. Reliability of the FuglMeyer assessment of sensorimotor recovery following cerebrovascular accident. Physical Therapy. 1983; 63(10):1606610. PMID: 6622535

[12] Smith YA, Hong E, Presson C. Normative and validation studies of the Nine-hole Peg Test with children. Perceptual and Motor Skills. 2000; 90(3):823-43. doi: 10.2466/ pms.2000.90.3.823

[13] Grice KO, Vogel KA, Le V, Mitchell A, Muniz S, Vollmer MA. Adult norms for a commercially available Nine Hole Peg Test for finger dexterity. American Journal of Occupational Therapy. 2003; 57(5):570-73. doi: 10.5014/ajot.57.5.570

[14] Cramer SC. Repairing the human brain after stroke: I. Mechanisms of spontaneous recovery. Annals of Neurology. 2008; 63(3):272-87. doi: 10.1002/ana.21393

[15] Krakauer JW. Motor learning: its relevance to stroke recovery and neurorehabilitation. Current opinion in Neurology. 2006; 19(1):84-90. doi: 10.1097/01.wco.0000200544.29915.cc

[16] Kwakkel G, Kollen B. Predicting activities after stroke: what is clinically relevant? International Journal of Stroke. 2013, 8(1):25-32. doi: 10.1111/j.1747-4949.2012.00967.x

[17] Tombaugh TN, McIntyre NJ. The mini-mental state examination: a comprehensive review. Journal of the American Geriatrics Society. 1992; 40(9):922-35. doi: 10.1111/j.15325415.1992.tb01992.x

[18] Murphy MA, Danielsson A, Sunnerhagen KS. Letter by Murphy et al. Regarding Article, Fugl-Meyer Assessment of Sensorimotor. Scandinavian Journal of Rehabilitation Medicine. 1975; 7:13-31.

[19] Kellor M, Frost J, Silberberg N, Iversen I, Cummings R. Hand strength and dexterity. American Journal of Occupational Therapy. 1971; 25(2):77-83. PMID: 5551515

[20] Domholdt E. Physical therapy research: principles and applications. Philadelphia: WB Saunders Company; 2000.

[21] Chou CY, Chien CW, Hsueh IP, Sheu CF, Wang CH, Hsieh CL. Developing a short form of the Berg Balance Scale for people with stroke. Physical Therapy. 2006; 86(2):195-204. PMID: 16445333

[22] Sveen U, Bautz-Holter E, Margrethe Sodring K, Bruun Wyller T, Laake K. Association between impairments, self-care ability and social activities 1 year after stroke. Disability and Rehabilitation. 1999; 21(8):372-77. doi: 10.1080/096382899297477
[23] Franceschini M, La Porta F, Agosti M, Massucci M. Is health-related-quality of life of stroke patients influenced by neurological impairments at one year after stroke? European Journal of Physical and Rehabilitation Medicine. 2010; 46(3):389-99.

[24] Morris JH, van Wijck F, Joice S, Donaghy M. Predicting health related quality of life 6 months after stroke: the role of anxiety and upper limb dysfunction. Disability and Rehabilitation. 2013; 35(4):291-99. doi: 10.3109/09638288.2012.691942

[25] Rosewilliam S, Roskell CA, Pandyan A. A systematic review and synthesis of the quantitative and qualitative evidence behind patient-centred goal setting in stroke rehabilitation. Clinical Rehabilitation. 2011; 25(6):501-14. doi: $10.1177 / 0269215510394467$

[26] Bear-Lehman J, Abreu BC. Evaluating the hand: issues in reliability and validity. Physical Therapy. 1989; 69(12):1025033. PMID: 2685841

[27] See J, Dodakian L, Chou C, Chan V, McKenzie A, Reinkensmeyer DJ, et al. A standardized approach to the Fugl-Meyer assessment and its implications for clinical trials. Neurorehabilitation and Neural Repair. 2013; 27(8):732-41. doi: $10.1177 / 1545968313491000$

[28] Lin JH, Hsu MJ, Sheu CF, Wu TS, Lin RT, Chen CH, et al. Psychometric comparisons of 4 measures for assessing upper-extremity function in people with stroke. Physical Therapy. 2009; 89(8):840-50. doi: 10.2522/ptj.20080285

[29] Page SJ, Levine P, Hade E. Psychometric properties and administration of the wrist/hand subscales of the Fugl-Meyer Assessment in minimally impaired upper extremity hemiparesis in stroke. Archives of Physical Medicine and Rehabilitation. 2012; 93(12):2373-376. doi: 10.1016/j.apmr.2012.06.017

[30] Sullivan KJ, Tilson JK, Cen SY, Rose DK, Hershberg J, Correa A, et al. Fugl-meyer assessment of sensorimotor function after stroke standardized training procedure for clinical practice and clinical trials. Stroke. 2011; 42(2):427-32. doi: 10.1161/strokeaha.110.592766

[31] Sanford J, Moreland J, Swanson LR, Stratford PW, Gowland C. Reliability of the Fugl-Meyer assessment for testing motor performance in patients following stroke. Physical Therapy. 1993; 73(7):447-54. PMID: 8316578

[32] Michaelsen SM, Rocha AS, Knabben RJ, Rodrigues LP, Fernandes CG. Translation, adaptation and inter-rater reliability of the administration manual for the Fugl-Meyer assessment. Brazilian Journal of Physical Therapy. 2011; 15(1):80-88. doi: 10.1590/s1413-35552011000100013

[33] Platz T, Pinkowski C, van Wijck F, Kim IH, Di Bella P, Johnson G. Reliability and validity of arm function assessment with standardized guidelines for the Fugl-Meyer Test, Action Research Arm Test and Box and Block Test: a multicentre study. Clinical Rehabilitation. 2005; 19(4):404-11. doi: 10.1191/0269215505cr832oa

[34] Karimi E, Kalantary M, Shafiee Z, Tabatabaiee M. Interrater reliability of the action research arm test and the upperlimb section of fugl-meyer test in adults with cva who were resident in Qazvin, Iran. Journal of Research in Rehabilitation Sciences. 2014; 10(1):76-76.

[35] Lundquist CB, Maribo T. The Fugl-Meyer assessment of the upper extremity: reliability, responsiveness and validity 
of the Danish version. Disability and Rehabilitation. 2016: 1-6. doi: 10.3109/09638288.2016.1163422

[36] Wang YC, Magasi SR, Bohannon RW, Reuben DB, McCreath HE, Bubela DJ, et al. Assessing dexterity function: a comparison of two alternatives for the NIH Toolbox. Journal of Hand Therapy. 2011; 24(4):313-21. doi: 10.1016/j. jht.2011.05.001

[37] Lee P, Liu CH, Fan CW, Lu CP, Lu WS, Hsieh CL. The test-retest reliability and the minimal detectable change of the Purdue Pegboard Test in schizophrenia. Journal of the Formosan Medical Association. 2013; 112(6):332-37. doi: 10.1016/j.jfma.2012.02.023

[38] Amirjani N, Ashworth NL, Olson JL, Morhart M, Chan KM. Validity and reliability of the Purdue Pegboard Test in carpal tunnel syndrome. Muscle \& Nerve. 2011; 43(2):171-77. doi: 10.1002/mus.21856

[39] Gallus J, Mathiowetz V. Test-retest reliability of the Purdue Pegboard for persons with multiple sclerosis. American Journal of Occupational Therapy. 2003; 57(1):108-11. doi: 10.5014/ajot.57.1.108

[40] Poole JL, Burtner PA, Torres TA, McMullen CK, Markham A, Marcum ML, et al. Measuring dexterity in children using the Nine-hole Peg Test. Journal of Hand Therapy. 2005; 18(3):348-51. doi: 10.1197/j.jht.2005.04.003

[41] Backman C, Gibson SCD, Parsons J. Assessment of hand function: the relationship between pegboard dexterity and applied dexterity. Canadian Journal of Occupational Therapy. 1992; 59(4):208-13. doi: 10.1177/000841749205900406 